\title{
Application of fuzzy logic in order to plan and create software for converting sound to text
}

\author{
Hiwa Hasanzadeh, Jafar Majidpour
}

Department Computer Science, Raparin University Rania, Iraq

\begin{tabular}{l} 
Article Info \\
\hline Article history: \\
Received Aug 21, 2019 \\
Revised Nov 12, 2019 \\
Accepted Feb 18, 2020 \\
\hline
\end{tabular}

Keywords:

Algorithm Analytical

Fuzzy logic

Neural network

Speed of converting sound to text

The challenge of software

\begin{abstract}
The challenge of software in the field of converting sound to text is that some words have different meanings. In these situations, the functions of each one is distinguished by the use of fuzzy logic and division of the working part of the work. In this regard, the words must first be classified according to the specialized discipline. In the second place, the principles of writing should be maintained. In this regard, the points and commands and the alignment of the sentence are all items that should be considered in the software. The next item is the volume and speed of word processing and the lack of restrictions in the number of languages used. But the time problem is very important. In this regard, the use of existing codes and the optimization and remedy of the problems is a suitable method. In this research, some issues including security and maintenance of software proprietary rights, and the speed of converting sound to text and reducing noise and optimal performance of software to convert audio to text based on software method and the principles of fuzzy logic and neural network were investigated. Results showed improvement algorithm Analytical and results outcomes.
\end{abstract}

This is an open access article under the $\underline{C C B Y-S A}$ license.

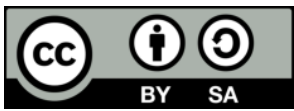

\section{Corresponding Author:}

Hiwa Hasanzadeh,

Department Computer Science,

Raparin University Rania, Iraq.

Email: HasanzadehH547@gmail.com

\section{INTRODUCTION}

In this review the topic of application of fuzzy logic in order to plan and create software for converting sound to text has been researched. The fuzzy logic first appeared in the new computing scene for the first time in setting the theory of fuzzy sets by [1]. The use of this section in software science can be simply defined as follows: fuzzy logic of the logic of the values of "zero and one" classical software goes further and opens a new door to the world of computer science and software because it uses and floats the space between zero and one in logic and argument itself. The fuzzy logic of the space between the two values is "go" or "do not go", new values "maybe we go" or "go if we" or even "is likely to go" extracts and uses. Thus, for example, after looking at a business account of a businessman, a banker can go beyond the logic of "lending" or "lending" and says: "We give a loan if ..." or "we do not give a loan but ...". The knowledge required for many of the issues studied appear in two distinct ways:

- Objective knowledge, such as models and equations and mathematical formulas that are pre-set and used to solve common problems of physics, chemistry, or engineering.

- $\quad$ Personal knowledge, such as knowledge that is partly descriptive and linguistic expression, but it is not usually possible to quantify them with the help of traditional mathematics. This kind of knowledge is tacit knowledge or implicit knowledge [2-10]. 
Since both types of knowledge are needed in practice, fuzzy logic tries to coordinate them in a regular, logical, and mathematical manner. The fuzzy logic did not go out of the university over the twenty years since 1965, because no one has the meaning He understood it. In the mid-80s of the last century, Japanese artisans realized the meaning and value of this science and applied fuzzy logic. Their first project was the design and control of the entire underground trains of the city of Sinda, which was planned and built by Hitachi. The result of this impressive Japanese design is simply summarized as follows: the start of the tragic impulse, subtle acceleration, braking and subtle standing and saving power. Subsequently, fuzzy logic was found to be very fast in the technology of Japanese audio and video devices (including nullifying the digital image while shaking hands with the cinematographer). Europeans began to use industrial use very late in the mid-1990s, after lagging behind the waves of scientific debate on fuzzy logic. Fuzzy logic is a multidirectional region and relies on the theory of fuzzy sets. The fuzzy sets themselves derive from the generalization and expansion of deterministic sets in a natural way.

Conclusive collections: definitive sets are in fact ordinary collections that are introduced at the beginning of the classical theory of collections. Adding a definite attribute in fact creates a distinction that helps to easily create one of the most innovative and vital concepts in fuzzy logic called membership function. In the case of definitive sets, the membership function has only two values in its range (in mathematics, the board of a function is the same as the set of all outputs of the function). Fuzzy sets are the generalization of the classical theory of collections that are used in fuzzy logic. The theory of these sets was invented by Lotf Ali Askarzadeh.

Language variables: linguistic variables are called variables that are acceptable values for them instead of numbers, words and sentences of human or machine languages. As mathematical calculations of numeric variables are used, fuzzy logic also uses language variables (spoken or non-numeric) It turns out. Language variables are expressed on the basis of language (spoken) values that are in the phrase set (words/ terms). Linguistic terms are attributes for linguistic variables. For example: The language variable "age" can be a set of phrases such as "adolescent", "young", "middle age" and "elderly" depending on the desired divisions of the person and the conditions.

The uncertainty trait appears in various forms in all fields and phenomena irrespective of the methodology used for studying, designing, and controlling. There is a lot of misunderstanding around us that we express them in everyday terms in terms of expressions. For example: "The weather is good," there is no amount of good air to measure it accurately, but it is a qualitative sense. In fact, the human brain defines sentences with various factors based on deductive thinking, and their modeling in mathematical language and formulas, if not impossible, will be very complex. Fuzzy logic is a new technology that replaces the methods used to design and model a system requiring complex mathematics and advancements, using linguistic values and expert knowledge [11-15].

To effectively tackle the increasing complexity of studying, studying, modeling and solving new problems in physics, engineering, medicine, biology and many other things, the creation and development of new computational methods have been developed that are more advanced in the ways of thinking and self-education of mankind to be close. The main objective is to enable computers as much as possible to ascertain and solve the very complex scientific problems with the same ease and simplicity that the human mind is capable of understanding and making quick and appropriate decisions. In the real world, many concepts A person understands and applies fuzzy (inaccurate, vague and vague). For example, though words and concepts such as hot, cold, long, short, old, young, and so on, do not refer to the exact number, but the human mind quickly and flexibly understands everyone, and in their decisions and conclusions uses. This is while the machine only understands the numbers and is precise. The purpose of the new methods in computer science is to firstly learn the mysteries of these abilities from humans and then to mention them as far as possible to the machine. Past scientific rules in Newtonian physics and mechanics are based on old logic. In old logic, we have only two states: white and black, yes and no, bright and dark, one and zero, true and false. Variables in nature or in calculations are of two types: the quantitative values that can be expressed by a certain number and the values. The qualities expressed on the basis of a characteristic. These two values can be converted. For example, in the case of people's height, if they are measured by numerical values (centimeters) and divided into tall and tall categories, and in this category, the threshold is 180 centimeters for height height, then all people under 180 centimeters are short on old logic. Even if the height of a person is $179 \mathrm{~cm}$, but in the fuzzy set, each of these attributes is defined on the basis of the membership function and is valued between zero and one. Since our minds do their work with other logic and make decisions, to start it is necessary to create and innovate a new and multi-resource area that fuzzy logic is one of them. Since our minds do their work with other logic and make its decisions, to start, create and invent the new and multi-value area needed that fuzzy logic is one of them [16-22]. 
Industrial applications: for each agenda and request for mechanical, electromagnetic or software operation, for which there is no absolute mathematical formula or instruction, and especially when the agenda is created by sentences, fuzzy logic software is robust and efficient. Some applications include:

- The guidance and control of any dynamic and moving device and installation can be best suited for fuzzy logic. Including washing machines, trains, Ibiza car brakes, elevators, cranes, conveyor belts, combustion engines, automatic take-offs and so on.

- $\quad$ Audio/visual digital devices.

- $\quad$ "Prospective" software to prevent hacking servers, controlling search engines on the Internet, translation software systems, robotics and artificial intelligence, examining the possibility of Serendipity impressions, medical engineering including pathology or CT scan, CCU, and ICU, heart pacemaker.

- $\quad$ Risk analysis, statistical and banking assessment for managers' decisions.

- Statistical calculations of insurance to find risk factors in contracts. (Many insurance companies in the world have been evaluating damage and customer compensation for a number of years by fuzzy software, and this way they are struggling with fraud and customer fraud) [23-28].

Fuzzy logic properties: over 50 years since the invention of Dr. Lotfi Zadeh in fuzzy logic, many articles have been written under his supervision or by referring to the original article, and various aspects of this logic and calculus have been studied based on fuzzy numbers. In this paper, we introduce some of the characteristics of fuzzy logic.

- $\quad$ Flexible implementation and simplicity of algorithms in "machine learning" methods

- $\quad$ The possibility of simulating logic and human thinking

- $\quad$ Possibility to create two solutions or an answer for an issue

- $\quad$ Suitable for solving problems with approximate answers

- $\quad$ A process look to inference with the use of flexible terms and conditions in fuzzy logic

- $\quad$ Ability to create nonlinear functions with arbitrary complexity

- There is a strong dependence of the researcher on the creation of fuzzy logic models

It is clear that fuzzy logic can not be considered as a comprehensive solution to all issues. So it's important to know when fuzzy logic should not be used. In the list below, we will introduce the fields that fuzzy logic should not be used.

- $\quad$ If the data and the results space are not suitable for fuzzy logic.

- $\quad$ Fuzzy logic is not working when used with five-dimensional senses.

- If fuzzy logic control and decision-making methods work well, then they do not need to be converted into fuzzy logic.

Fuzzy logic structure: fuzzy logic has four main sections that are presented below:

Basic rules: This section includes all the rules and conditions that are designated as "if ... then" by an expert to be able to control the decisions of a "decision-making system." Given the new methods in fuzzy theory, it is possible to adjust and reduce rules and rules so that the best rules can be achieved with the least rules.

Fuzzification: in the phase of phasing, the inputs are converted to fuzzy information. This means that the numbers and information to be processed should be converted to fuzzy sets and numbers. The input data, which is measured by sensors in a control system, for example, is modified in this way and is prepared for processing based on fuzzy logic.

Inference intelligence: in this section, the degree of adaptation of inputs from fuzzification is determined by the base rules. in this way, based on the percentage of adaptation, different decisions are made as the results of the fuzzy inference engine.

Returning from fuzzy: at the last stage, the results of the fuzzy inference, which are fuzzy sets, transform into quantitative and quantitative data and information. At this point, you decide on the best choices for outputs that include different decisions, along with percentages for different adjustments. Usually, this choice will be based on the highest degree of compliance.

Fuzzy logic and probability: as stated above, in a fuzzy logic the degree of certainty or the correctness of a proposition is expressed by a number at 0 to 1 . In the theory of probability, we use a number between 0 and 1 for the occurrence of an event. In this way, there seems to be an adaptation between these two concepts and, of course, with different uses. So, if we can say with the view of theory: "With a $90 \%$ probability, a person is glasses." It can be seen in fuzzy logic: "The degree of individual membership to the group of eyewitnesses is 0.9." Thus, probability is a mathematical model for unspecified and random phenomena. On the other hand, fuzzy logic is also a mathematical model used to determine the truth for any phenomenon, using a value as the "correctness".

The advantages of using fuzzy logic systems: the structure of fuzzy logic systems is simple and understandable. Fuzzy logic is also widely used today on a commercial and laboratory scale. On the other hand, it is possible to control better and more efficient cars and save costs by using fuzzy logic. 
Fuzzy logic may be subtracted from fuzzy results, but due to the acceptability of the results, it can be used reliably, especially if we are faced with inadequate inputs.

Also, in the field of control, fuzzy logic can be programmed in such a way that the process of production is not stopped by the failure of the sensors. In this context, it is possible to increase the efficiency of systems with fuzzy logic, so that using inexpensive sensors, the process control system can be done at a very low cost. In the end, perhaps the best reason to use fuzzy logic could be solved by solving complex problems with more effective and simpler solutions.

Disadvantages of fuzzy logic systems: because of the rule of law, the decision-making process is based on fuzzy logic; if these rules are flawed or shaped, the results may not be acceptable at all. Selecting a membership function and basic rules is one of the most difficult parts of creating fuzzy systems. On the other hand, the implementation of fuzzy logic in common hardware requires many and time-consuming experiments. Unfortunately, the efficiency of fuzzy logic is less in recognizing the pattern than the neural network in machine learning. That's why it's less at data science.

\section{METHODOLOGY}

MATLAB is a software environment for numerical computing and a fourth-generation programming language. The word MATLAB also means the digital computing environment, and also the meaning of its own programming language, which is composed of two MATRIX (matrix) and LABoratory (lab) combinations. This name implies a program-oriented matrix approach, in which even single numbers are considered as matrices. Working with matrices in MATLAB is very simple. In fact, all data in MATLAB is stored in the form of a matrix. For example, a number (scalar) is stored in the form of a $1 * 1$ matrix. A string like "Whale is the largest animal" is stored in a matrix with a single row and several columns (the number of columns is the number of characters). Even an image is stored in the form of a 3D matrix whose first and second dimension is used to determine the coordinates of the points and the third dimension is used to determine the color of the points. The audio files are also stored in MATLAB in the form of single column matrices (column vectors); so it's no surprise that MATLAB is the acronym for the matrix lab. In addition to the many functions it owns, the programmer can also define new functions. Creating a graphical user interface Like dialogues that exist in visual environments such as BASIC and C, it is possible to do this in MATLAB. This feature provides better connectivity between applications written by MATLAB and users. Mometx, a product of the Metrox company, has many uses for various groups of engineers such as electrical engineering, mechanics, computers, etc. Cleve Moler, Head of Division Computer Science at the University of New Mexico began developing MATLAB in the late 1970s. He designed the program to allow his students to access LINPACK and EISPACK without having to learn Fortran. This topic soon expanded to other universities and became an interest audience in the applied mathematical community. Engineer Jack Lowell met with Mattalb during a meeting with Moler from Stanford University in 1983. He decided to collaborate with Moler, with the recognition of the business potential of MATLAB. They published MATLAB in 1984 and founded MathWorks in 1984. In the year 2000, MATLAB was rewritten to use a newer library collection to manipulate the matrix. MATLAB was first used by researchers and practitioners in engineering control, Little's expertise, but quickly expanded in many areas. Also, in education, in particular linear algebra training, numerical analysis and image processing are used. The company was founded in 1984 and now has more than 2000 personnel. The company's headquarters are located in Natick, Massachusetts, USA.

\section{RESULTS}

The MATLAB core has been written in $\mathrm{C}$ for speed and performance, but its graphical interface is implemented in Java. Matthew's apps are mostly open source and in fact MATLAB (like BASIC) is a commentator (computer), not a compiler. The power of MATLAB is due to its flexibility and ease of operation, as well as the company and various groups, including universities around the world, and some engineering companies annually add special-utility tools that enhance its efficiency and popularity. The list of these toolbars is as follows:

a. Simulink, a tool for simulating single systems

b. Metabel telecommunications toolbox, telecommunications engineering functions and tools

c. Metabel control toolbox, control functions and calculating tools

d. Fuzzy metabel toolkit, functions and fuzzy computing tools

e. MATLAB calculator tool, numerical calculator functions and tools

f. Toolkit for estimating mathematics, functions and computing tools. System estimation in control engineering 
g. Metabolic toolkit, functions and statistics tools

h. Toolbar for collecting data from MATLAB, functions and data collection tools

i. MATLAB neural network tools, functions and tools for calculating neural network

j. $\quad$ Picture tools for image processing, functions and image processing tools

k. Metabolic sound processing tools, functions, and audio processing tools

1. The MATLAB probability tool

m. Symbolic calculator tool

n. Realtime workshop toolkit, Realtime system calculations functions and tools [3, 4]

The following code is used to process sound in MATLAB software:

[road,fs]=wavread('road.wav'); \% loads "the long and winding road" clip

The road array contains audio data and fs sampling rate. Sampling rate for a similar music CD ( $\mathrm{fs}=44,100$ samples / second). The array size is expressed as:

size(road)

Separating the right and left channel signals is done according to the following code:

left=road(:,1);

right=road $(:, 2)$;

The display of the data chart against time is based on the following commands:

time $=(1 / \mathrm{fs}) *$ length $($ left $)$;

$\mathrm{t}=$ linspace $(0$, time,length(left));

plot(t,left)

xlabel('time (sec)');

ylabel('relative signal strength')

Figure 1 shows the workspace box detail. As it can be seen in Figure 1, the sampling frequency is 44100 .

\begin{tabular}{|c|c|c|c|c|}
\hline \multicolumn{5}{|l|}{ Workspace } \\
\hline 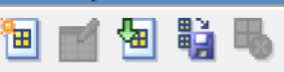 & Stack: purenouse - & QP Select data to pl & & $*$ \\
\hline Name - & Value & Min & Max & \\
\hline$\boxplus$ freq & 44100 & 44100 & 44100 & \\
\hline perfectSound & $<606171 \times 2$ double $>$ & $<$ Too many ... & $<$ Too ... & \\
\hline
\end{tabular}

Figure 1. Workspace box detail

For example, a portion of the audio data is displayed as follows :

time $=(1 / 44100) * 2000$;

$\mathrm{t}=$ linspace $(0$,time, 2000);

$\operatorname{plot}(\mathrm{t}, \mathrm{left}(1: 2000))$

xlabel('time (sec)');

ylabel('relative signal strength');

Sound playback :

soundsc(left,fs) \%

plays left channel as mono soundsc(right,fs) \%

plays right channel mono sound nearly the same) soundsc(road,fs) \%

plays stereo

Sound function :

- sound $(\mathrm{y}, \mathrm{Fs})$

-sound(y,Fs, bits)

- Convert Matrix to Audio

- Send y audio signal to speaker with Fs sampling rate

- For single-channel voices y is a m-by-1 matrix ( $\mathrm{m}$ is the number of audio samples)

- y can be a matrix of m-by-2 matrix. The first column is the left channel and the second is the right channel channel

The sound function assumes that the values of $\mathrm{y}$ are between 1 and 1 , and eliminates values outside of this range.

Increase or decrease the volume based on the following code :

Application of fuzzy logic in order to plan and create software for converting sound to... (Hiwa Hasanzadeh) 
$\%$ make sound louder/quieter clear all;

$1 . \mathrm{Fs}=44100 ; \%$ sampling frequency

2.dur $=1 ; \%$ duration of sound (in sec)

$3 . \%$ time vector

$4 . \mathrm{t}=[0: 1 / \mathrm{Fs}:$ dur-1/Fs $] ; \% 1$ second $-->$ length $(\mathrm{t})=44100$

$5 . \%$ frequency

6. freq $=440$

7.f $=\sin (2 *$ pi $*$ freq $* \mathrm{t})$

8. $\% \% \% \% \% \% \% \% \% \% \% \% \% \% \% \% \% \% \% \% \%$

$9 . \%$ scale sound

$10 . \mathrm{amp}=.5$;

11.f_amp $=$ amp $*$ f;

12.sound(f_amp,Fs)

13.plot( $t(1: 2 *$ round(1/freq*Fs $)), f(1: 2 * \operatorname{round}(1 /$ freq*Fs $)))$

14.hold on

15.plot( $t(1: 2 *$ round(1/freq*Fs $)), \mathrm{f}$ amp $(1: 2 * \operatorname{round}(1 /$ freq*Fs $))$, 'r'

Finally, the output is in the form of a diagram (2):

Sine wave based audio signal as shown in Figure 2.
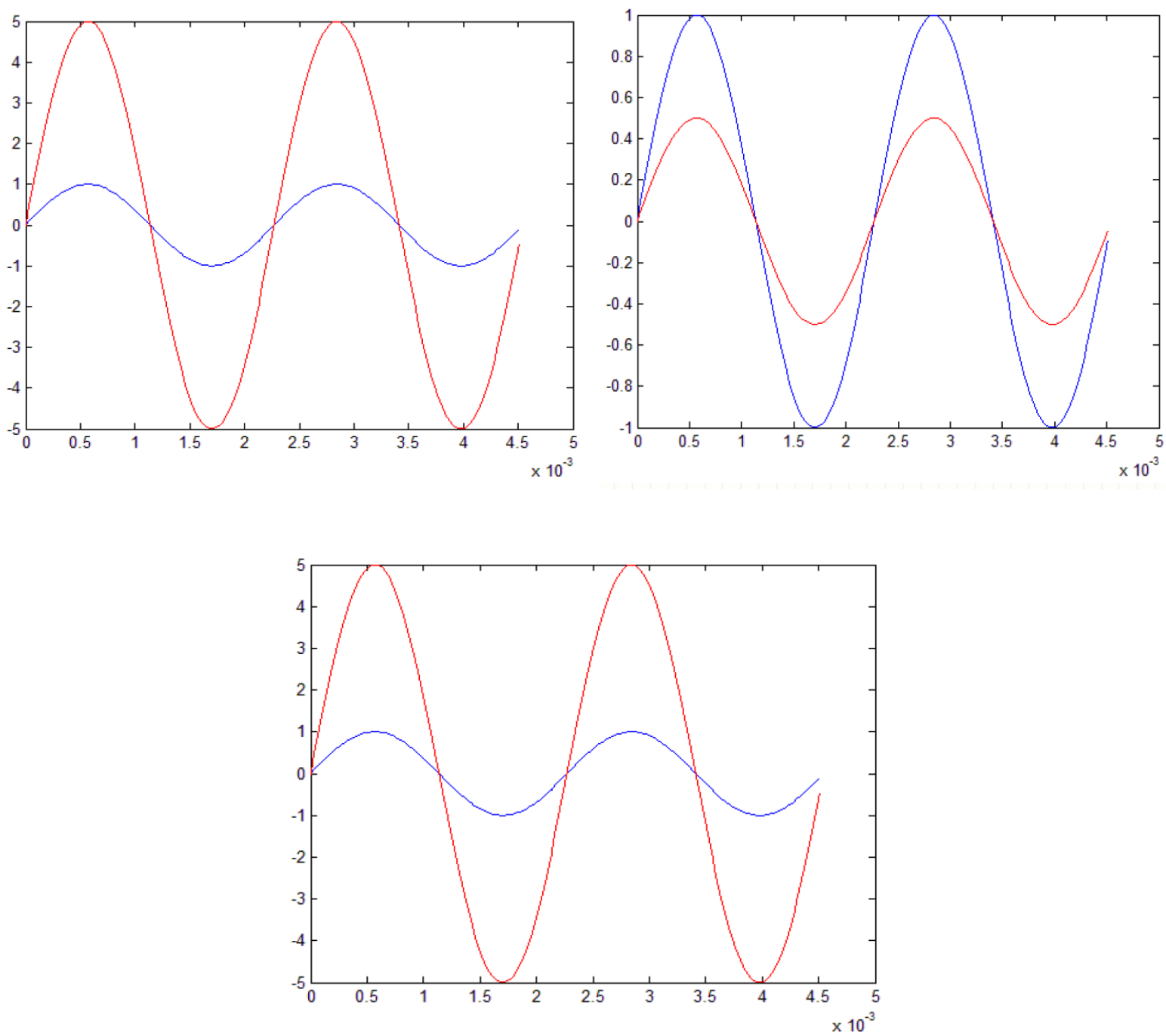

Figure 2. Sine wave based audio signal 
Sound processing in MATLAB:

soundsc(left,fs); \% Original left channel

soundsc(left-right,fs);

soundsc(hootie(:,1),fs); \%

Original left channel

soundsc(hootie(:,1)-hootie(:,2),fs);

[hootie,fs]=wavread('hootie.wav'); \% loads Hootie out=hootie;

for $n=2$ :length(hootie)

$\operatorname{out}(n, 1)=.9 * \operatorname{out}(n-1,1)+$ hootie $(n, 1) ; \%$ left

$\operatorname{out}(\mathrm{n}, 2)=.9 * \operatorname{out}(\mathrm{n}-1,2)+$ hootie $(\mathrm{n}, 2) ; \%$ right

end

soundsc(hootie,fs) $\%$ original

soundsc(out,fs) \%

out=hootie;

for $\mathrm{n}=2$ :length(hootie)

out(n,1)=hootie (n,1)-hootie(n-1,1); \% left

$\operatorname{out}(\mathrm{n}, 2)=\operatorname{hootie}(\mathrm{n}, 2)-\operatorname{hootie}(\mathrm{n}-1,2) ; \%$ right

end

soundsc(out,fs) \% high pass filtered

Noise and add noise to the audio signal :

The function $\mathrm{B}=$ randn $(\mathrm{A})$ produces real random numbers such that the mean is zero and the standard deviation is one, which is the same noise that we need.

[perfectSound, freq] = wavread('road.wav');

$\mathrm{N}=\operatorname{randn}($ length(perfectSound), 2);

noisySound $=$ perfectSound $+\mathrm{N}$;

The challenge of software in the field of converting sound to text is sometimes the problems of recognizing and converting sound and words. Some words have different meanings and may have problems with the translation of the machine. In these situations, the functions of each one are distinguished by the use of fuzzy logic and division of the working part of the work. In this regard, the words must first be classified according to the specialized discipline. For example, for basic sciences and engineering and medicine, the meaning of a specific word can be exactly defined in each field, and the type of string is specified when entering data. The better the split, the better. In the second place, the principles of writing should be maintained. In this regard, the points and commas and the alignment of the sentence are all items that should be considered in the software. The next item is the volume and speed of word processing and the lack of restrictions in the number of languages used. But the time problem is very important as one of a variety of software developed by the algorithm is such that you can convert a 1-minute audio file to the text in 5 seconds. In this regard, the use of existing codes and the optimization and remedy of the problems is a suitable method. For example, audio cryptography is the process of capturing additional information in the host signal in order to prove ownership. The criterion of the soundness of sound cryptography, such as the hidden nature of the hidden watermark, is unobtrusive to unauthorized persons to prevent being removed, to be resistant to all signal processing attacks and to be revealed in order to prove ownership. The algorithm Voice caching is generally divided into two groups of time domain and conversion domain. Convergence domain algorithms are more robust against time-domain algorithms. But their resistance to signals where the number of components in their domain of transformation is very low is not satisfactory. In addition, these algorithms are more complicated and time-consuming than time domain algorithms. Time domain techniques generally use two strategies. The first method is to manipulate the signal samples in order to capture the watermark bit, also called the LSB bit encryption method, and the second method of adding subtle noise, or the watermark signal, which carries the message to the host signal (the wide spectrum methods), Hide delay and so on. It can be said that almost all time-domain algorithms use the properties of the human auditory system to obscure the injectable noise. Images from the text output of the Text-to-Speech project are shown in the following Figure 3. In this regard, the use of fuzzy logic can identify their values and parameters by identifying their respective variables and parameters, and refute the best way to improve the results. In fact, the sample outputs shown in the following are based on a combination of the MATLAB code and the fuzzy logic method, aimed at optimizing the software. Figure 3 shows screenshots of text output in sound. For example, as it can be seen the msg_bin is a matrix with $18 \times 8$ dimension. 


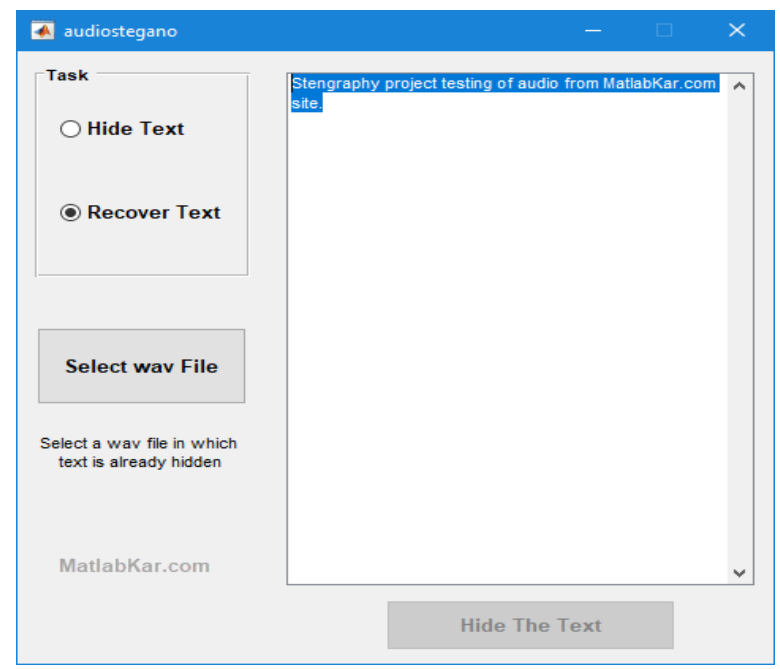

\begin{tabular}{|c|c|}
\hline Workspace & () \\
\hline Name - & Value \\
\hline$\theta$ ans & 0 \\
\hline$\#$ count & 89830 \\
\hline \# data_size & 179660 \\
\hline D dta & $89830 \times 1$ double \\
\hline fid 1 & 3 \\
\hline$\#$ fid2 & 3 \\
\hline$a b c$ filename & 'audio.wav' \\
\hline $\mathrm{abc}$ header & $40 \times 1$ char \\
\hline$\#$ identity & {$[1,0,1,0,1,0,1,0]$} \\
\hline Ien & 144 \\
\hline Ien_bin & $20 \times 1$ double \\
\hline Isb & $1 \quad$ MatlabKar.com \\
\hline m $\mathrm{m}$ & 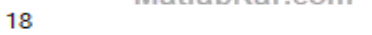 \\
\hline$\#$ m_bin & {$[0 ; 1 ; 0 ; 0 ; 1 ; 0 ; 0 ; 0 ; 0 ; 0]$} \\
\hline $\mathrm{abc} \mathrm{msg}$ & 'Hello how are you?' \\
\hline \# msg_bin & $18 \times 8$ double \\
\hline Hsg_bin_re & $144 \times 1$ double \\
\hline Hsg_double & $1 \times 18$ double \\
\hline 其 & 8 \\
\hline n_bin & {$[0 ; 0 ; 0 ; 1 ; 0 ; 0 ; 0 ; 0 ; 0 ; 0]$} \\
\hline pathname & 'C: $\backslash$ project\' \\
\hline secc secmsg & 'Hello how are you?' \\
\hline Hecmsg_bin & $144 \times 1$ double \\
\hline H secmsg_bin_re & $18 \times 8$ double \\
\hline H secmsg_double & $18 \times 1$ double \\
\hline
\end{tabular}

\begin{tabular}{|c|c|}
\hline Workspace & C \\
\hline Name - & Value \\
\hline$\boxplus$ ans & 0 \\
\hline$\theta$ count & 89830 \\
\hline \# data_size & 179660 \\
\hline Odta & $89830 \times 1$ double \\
\hline \# fid1 & 3 \\
\hline$\#$ fid2 & 3 \\
\hline$a b c$ filename & 'audio.wav' \\
\hline$a b c$ header & $40 \times 1$ char \\
\hline identity & {$[1 ; 0 ; 1 ; 0 ; 1 ; 0 ; 1 ; 0]$} \\
\hline Mlen & 144 \\
\hline Mlen_bin & $20 \times 1$ double \\
\hline H Isb & MatlabKar.com \\
\hline$\theta \mathrm{m}$ & (1) \\
\hline m_bin & {$[0 ; 1 ; 0 ; 0 ; 1 ; 0 ; 0 ; 0 ; 0 ; 0]$} \\
\hline $\mathrm{abc} \mathrm{msg}$ & 'Hello how are you?' \\
\hline \#sg_bin & $18 \times 8$ double \\
\hline \#sg_bin_re & $144 \times 1$ double \\
\hline msg_double & $1 \times 18$ double \\
\hline$\boxplus n$ & 8 \\
\hline n_bin & {$[0 ; 0 ; 0 ; 1 ; 0 ; 0 ; 0 ; 0 ; 0 ; 0]$} \\
\hline$a b c$ pathname & 'C:\project \' \\
\hline
\end{tabular}

Figure 3. Screenshots of text output in sound

\section{CONCLUSION}

In this paper, the use of fuzzy logic was considered and designed to program and create software for converting sound to text. The fuzzy logic first appeared in the new computing scene for the first time to set up the theory of fuzzy sets by Professor Lotfizadeh (1965). The term "fuzzy" means dull and unspecified. In ordinary life, we sometimes encounter situations where we do not know what the right or wrong decision is and the proper functioning of our eyes is hidden. At this time, fuzzy logic offers a logical and valuable proposition. Thus, for each situation, the uncertainty level can be determined. For this reason, sometimes fuzzy logic is also a disagreeable logic because the results are created with suspicion. Fuzzy logic has now helped solve many decision-making issues, which in most cases produces the best input-based decision. Fuzzy logic is based on human decision-making, as can be attributed to the development of Aristotle logic or Boolean logic. Today, based on fuzzy logic, fuzzy numbers, fuzzy arithmetic, and even statistical fuzzy Tools, such as fuzzy clustering, have been created. Devices and computers that have a much better performance than Boolean logic devices. The challenge of software in the field of converting sound to text is sometimes the problems of recognizing and converting sound and words. Some words have different meanings and may have problems with the translation of the machine. In these situations, the functions of each one are distinguished by the use of fuzzy logic and division of the working part of the work. 
In this regard, the words must first be classified according to the specialized discipline. For example, for basic sciences and engineering and medicine, the meaning of a specific word can be exactly defined in each field, and the type of string is specified when entering data. The better the split, the better. In the second place, the principles of writing should be maintained. In this regard, the points and commas and the alignment of the sentence are all items that should be considered in the software. The next item is the volume and speed of word processing and the lack of restrictions in the number of languages used. But the time problem is very important as one of a variety of software developed by the algorithm is such that you can convert a 1-minute audio file to the text in 5 seconds. In this regard, the use of existing codes and the optimization and remedy of the problems is a suitable method. In this research, some issues including security and maintenance of software proprietary rights, and the speed of converting sound to text and reducing noise and optimal performance of software to convert audio to text based on software method and the principles of fuzzy logic and neural network were investigated. Results showed improvement algorithm Analytical and results outcomes.

\section{REFERENCES}

[1] J. M. Mendel, "Uncertain Rule-Based Fuzzy Logic Systems: Introduction and New Directions," Prentice Hall PTR, 2001.

[2] N. K. Kasabov, "Foundations of Neural Networks, Fuzzy Systems, and Knowledge Engineering," The Press, 1996.

[3] W. L Martinez, "Computational Statistics in MATLAB," Wiley Interdisciplinary Reviews, 2010.

[4] H. Karaca and R. Akkaya, "Modeling, Simulation and Analysis of Matrix Converter Using Matlab and Simulink," International Journal of Modeling and Optimization, vol. 2, no. 3, pp. 328-332, 2012.

[5] J. Schaeffer, "Didn't Samuel Solve That Game?," In: One Jump Ahead. Springer, Boston, MA, 2009.

[6] L. Shane and M. Hutter, "A Collection of Definitions of Intelligence," arXiv:0706.3639, pp. 06-25, 2007.

[7] S. R. Sutton and G. B. Andrew, "Reinforcement Learning: An Introduction," MIT Press, 1998.

[8] T. Hastie, R Tibshirani, J Friedman, "The Elements of Statistical Learning: Data Mining, Inference, and Prediction," Springer, 2009.

[9] G. Diet Erich, "Machine Learning Thomas," Department of Computer Science - Oregon State University.

[10] T. M. Mitchell, "Machine Learning," McGraw-Hill Companies, Inc., 1997.

[11] M. Beutnagel, et al., "The AT\&T Next-Gen TTS System," 137th Acoustical Society of America Meeting, pp. 18-24, 1999.

[12] W. Hamza1 and R. Donovann, "Data-Driven Segment Preselection in the IBM Trainable Speech Synthesis System," ICSLP, pp. 2609-2612, 2002.

[13] D. H. Klatt, "Linguistic Uses of Segmental Duration in English: Acoustic and perceptual Evidence," Journal of Acoustic Society of America, vol. 59, no. 5, pp. 1208-1221, 1976.

[14] A. K. Syrdal, et al., "Three Methods of the Intonation Modeling," The Third Workshop on Speech Synthesis, Australia, 1998.

[15] A. R. Ekti, "Fuzzy Logic Approach for Layered Architecture Cognitive Radio Systems," In International Telecommunications Conference, pp. 61-71, 2019.

[16] S. Gates, CL Prachyl and C. Sullivan, "Using report to the nations on occupational fraud and abuse to stimulate discussion of fraud in accounting and business classes," Journal of Business and Behavioral Sciences, vol. 28, no. 1, pp. 106, Apr 2016.

[17] W. Albrecht, C. C. Albrecht, C. O. Albreacht, "Fraud and corporate executives: Agency, Stewardship and Broken Trust," Journal of Forensic Accounting, vol. 5, pp. 109-130, 2011.

[18] P. R. Gillett, and N. Uddin, "CFO Intentions of Fraudulent Financial Reporting," Auditing, vol. 24, no. 1, pp. 55-57, 2005.

[19] S. Susanti, M. Andhani, S. Zulaihati, "The Influence of Intellectual Capital and Good Corporate Governance on Financial Performance in Banking Companies," AFEBI Accounting Review, vol. 3, no. 2, Jun 2019.

[20] I. J. Chen and A. Paulraj, "Understanding supply chain management: critical research and a theoretical framework," International Journal of production research, vol. 42, no.1, pp.131-163, 2004.

[21] M. Funk, "Human Resource and Training In Mental Health," World Health Organization, 2005.

[22] H. T. Nguyen, C. L. Walker, E. A. Walker, "A first course in fuzzy logic," CRC press, 2018.

[23] C. S. Saunders and J. W. Jones, "Measuring Performance of the Information System Function," Journal of Management Information System, vol. 8, no. 4, pp. 63-82, 1992.

[24] J. L. Chan, "International public sector accounting standards: conceptual and institutional issues," The Harmonization of Government, vol. 21, pp. 1-5, 2008.

[25] L. M. Spencer and M. S. Spencer, "Competence at Work Models for Superior Performance," John Wiley \& Sons.Inc, 1993.

[26] L. J. Mullins, "Management and Organization Behavior," Prentice Hall/Financial Time, 2005.

[27] Y. Ikegami, et al., "A Visual Counseling Agent Avatar with Voice Conversation and Fuzzy Response," In 2018 World Automation Congress (WAC), pp. 1-5, 2018.

[28] G. Felix, et al., "A review on methods and software for fuzzy cognitive maps," Artificial Intelligence Review, vol. 52, no.3, pp. 1707-1737, 2019. 


\section{BIOGRAPHIES OF AUTHORS}

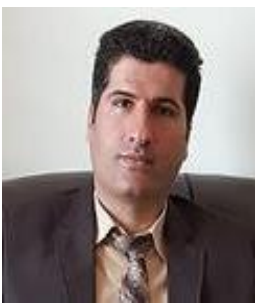

Hiwa Hassanzadeh was born in Mahabad, West Azerbaijan, Iran in 1983. He received his bachelor degree in Computer Sciences from Payam-E-Noor university (PNU), Mahabad, Iran in 2009. He received Master's degrees in Software Engineering from (PNU), Tehran, Iran in 2012. He has joined University of Raparin from Iraq as an Assistant lecturer in the Basic Education Department since 2013 and he has got lecturer degree in 2019. His primary research interests are machine learning, deep learning, signal processing, and improve algorithm.

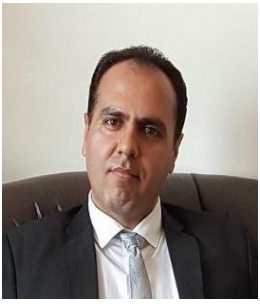

Jafar Majidpour was born in Mahabad, West Azerbaijan, Iran in 1982. He received his bachelor degree in Computer Software from Omid Nahavand Institute of Higher Educaation, Nahavand, Hamadan, Iran in 2009. He received Master's degrees in Software EngineeringSoftware from East Azarbaijan Science Research Branch, Tabriz, Iran in 2013. He has joined University of Raparin from Iraq as an Assistant lecturer in the Basic Education Department since 2013 and he has got lecturer degree in 2019. His primary research interests are machine learning, deep learning, image processing. 